\title{
Role of contrast-enhanced ultrasound in the evaluation of vascularization of hepatocellular carcinoma
}

\author{
Francesco Loria ${ }^{1}$, Giuseppe Loria ${ }^{2}$, Salvatore Basile ${ }^{1}$, Giuseppe Crea ${ }^{1}$, Luciano Frosina ${ }^{3}$, Francesca Frosina ${ }^{4}$ \\ ${ }^{1}$ Department of Radiology, PO Palmi, ASP 5 Reggio Calabria, 89015 Palmi, Italy \\ ${ }^{2}$ Department of Radiology, PO Lamezia Terme, ASP Catanzaro, 88046 Lamezia Terme, Italy \\ ${ }^{3}$ School of Medicine, University of Messina, 98124 Messina, Italy \\ ${ }^{4}$ School of Medicine, Bio-Medical Campus, 00128 Rome, Italy
}

Correspondence to: Dr. Francesco Loria, Department of Radiology, PO Palmi, ASP 5 Reggio Calabria, 89015 Palmi, Italy. E-mail: francescoloria956@alice.it

How to cite this article: Loria F, Loria G, Basile S, Crea G, Frosina L, Frosina F. Role of contrast-enhanced ultrasound in the evaluation of vascularization of hepatocellular carcinoma. Hepatoma Res 2016;2:316-22.

Article history:

Received: 04-07-2016

Accepted: 18-08-2016

Published: 22-11-2016

Key words:

Contrast-enhanced ultrasound, hepatocarcinoma, multislice computed thomography

\begin{abstract}
\end{abstract}
\section{INTRODUCTION}

Hepatocellular carcinoma (HCC) is the primitive, most common malignant tumor of the liver accounting for $70-84 \%$ of hepatic tumors. The early identification of $\mathrm{HCC}$ is very important in obtaining good therapeutic outcomes, thanks to several options such as percutaneous therapies, surgical resections and transplant. Ultrasound examination and measurements of the levels of alpha-fetus protein in serum are the main screening options. Several methods have been used to evaluate the intralesional vascularization of hepatic focal lesions..$^{[1-5]}$ Computed thomography (CT) is the second most common

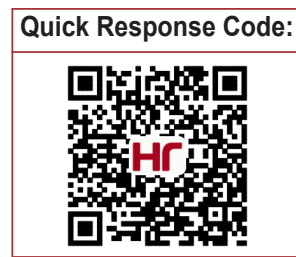


screening option.

The development of second-generation ultrasound contrast media and dedicated software has improved the diagnostic capabilities of ultrasound in the individualization and characterization of focal hepatic lesions, ${ }^{[6-10]}$ as it allows clinicians to study intralesional vascular architecture in real time in all contrastographic phases. ${ }^{[11-15]}$

Each contrastographic phase has its own specificity, useful in diagnosis; in particular, in the arterial phase, it is fundamental to evaluate the pattern and the grade of vascularization, while the portal and late phase are also useful for the correct diagnosis.

\section{METHODS}

Between January 2009 and May 2014, 67 patients affected by hepatocarcinoma, who presented an overall of 92 nodules, were examined and enrolled in the study. There were 23 females and 44 males with an average age of 68 years, of whom 62 presented a chronic liver disease, while 5 did not present any hepatic symptoms. The diagnosis of HCC was established by confirming the presence of a lesion, which assumed enhancement in the arterial phase with wash-out in portal and late phases. The same parameters were utilized in both contrast-enhanced ultrasound (CEUS) and in multislice CT (MSCT).

The three vascular phases were evaluated: arterial (0-35 s from the injection of the MdC), portal (35-90 s) and sinusoidal (from $90 \mathrm{~s}$ to approximately $6 \mathrm{~min}$ ). Increases in serum levels of alpha-fetoprotein were also evaluated.

\section{CEUS}

The examinations used GE LOGIQ 5 EXPERT and ESAOTE MY LAB equipment with a specific incorporated software designed to work at low mechanical index, with $3.5 \mathrm{MHz}$ convex transducers. The contrast medium in all cases was the SonoVue (Bracco, Italy), consisting of micro bubbles of stabilized phospholipids containing sulphur hexafluoride. This was injected as a bolus in an antecubital vein, followed by an injection of $10 \mathrm{~mL}$ of physiological solution. In no case was a second injection of MdC given. Before the intravenous injection, a basal echography of the liver was done in order to evaluate the most suitable ultrasound window to study the lesion. The identified lesion was studied "in real time" up to approximately 6 min from the injection and the enhancement was always compared with the surrounding parenchyma. All phases of the tests were registered on a compact disc (CD) to be evaluated again. In no case were complications manifested.

\section{MSCT}

The examinations used a CT multidetector scanner of GE light-speed (16 and 64 canals). All examinations were done in basal conditions and after intravenous injection of approximately $90-120 \mathrm{~mL}$ of $\mathrm{MdC}$, at a $4 \mathrm{~mL} / \mathrm{s}$ speed. Smart prep was always used for acquisition of the arterial phase.

\section{Analysis of the images}

The vascularization of the single lesion, using both CEUS and CT, was classified as hyper-, iso- and hypovascular in each one of the evaluated phases, always in relation to the enhancement of the condition of surrounding parenchyma.

\section{Statistical analysis}

Fisher's test was used to compare the results of CEUS with MSCT. Furthermore, the results of the vascularization comparing CEUS and MSCT were evaluated in relation to the site and the size of the lesions. A $P$-value of $<0.05$ was considered statistically significant.

\section{RESULTS}

In the arterial phase [Table 1], 75 of 92 nodules were hyperdense in MSCT; of these in using CEUS, 66 $(88 \%)$ were hypervascular [Figure 1] and $9(12 \%)$ were isovascular [Figure 2].

Eleven of 92 nodules were isodense in MSCT; of these using CEUS, 3 (27\%) were hypervascular [Figure 3], 8 (73\%) were isovascular. Six of 92 nodules were hyperdense in MSCT, using CEUS, $2(33 \%)$ were hypervascular, $4(67 \%)$ isovascular. Seventy-six of 92 nodules were localized at a depth not greater than $9 \mathrm{~cm}$ from the abdominal wall, 16 of 92 were localized at a greater depth [Table 2]. Of the 64 out of 76 nodules localized at a depth not greater than $9 \mathrm{~cm}$ that appeared hypervascular using MSCT, $61(95 \%)$ appeared hypervascular using CEUS. Of the

Table 1: Comparison between arterial phase seen with MSCT and early vascular phase seen with CEUS

\begin{tabular}{lccc}
\hline \multirow{2}{*}{ Arterial phase in MSCT } & \multicolumn{3}{c}{ Early vascular phase seen in CEUS } \\
\cline { 2 - 4 } & Hyper & Iso & Total \\
\hline Hypervascular & $66(88 \%)$ & $9(12 \%)$ & 75 \\
Isovascular & $3(27 \%)$ & $8(73 \%)$ & 11 \\
Hypovascular & $2(33 \%)$ & $4(67 \%)$ & 6 \\
Total & $71(77 \%)$ & $21(23 \%)$ & 92 \\
\hline
\end{tabular}

MSCT: multislice computed thomography; CEUS: contrast enhanced ultrasound 

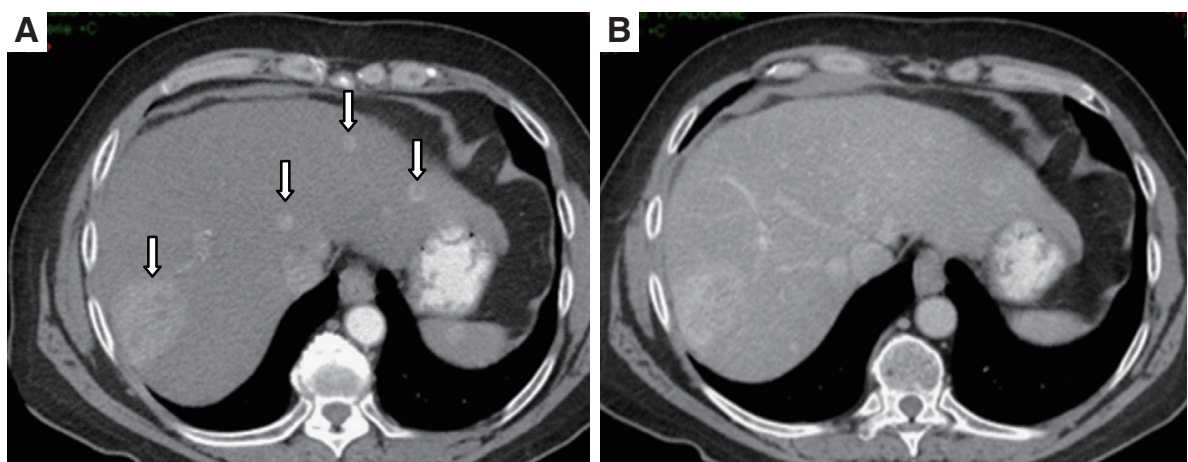

Figure 1: Arterial phase $(A, C)$ and portal phase $(B, D)$ seen in MSCT and CEUS, in the same patient with multifocal hepatocarcinoma. CEUS (C, D) appears in a double image: $B$ mode on the left and contrast enhancement on the right. Both modalities allow a good evaluation of the hypervascularization of the lesions in arterial phase (arrows) and the washout in the portal phase. MSCT allows the same scan in the arterial phase (A) to show 4 nodules. CEUS, in its windows in the same phase $(C)$ shows only 2 nodules. CEUS allows an extremely
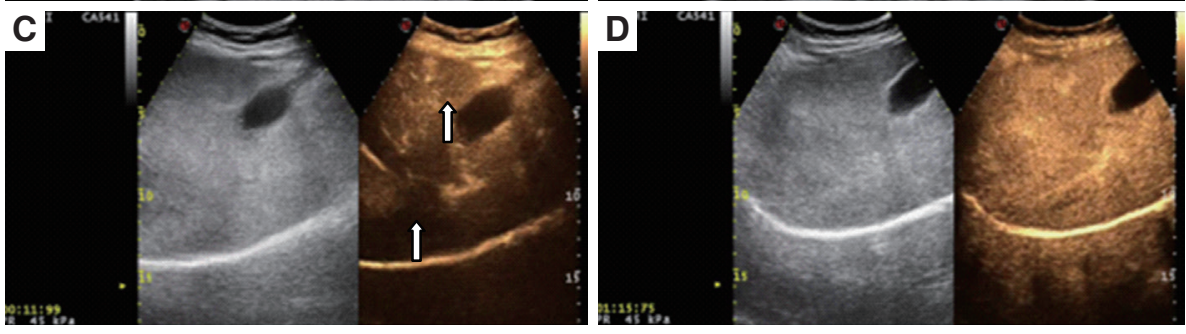
rapid scan of the arterial phase $(C)$ with enhancement of lesions after $11 \mathrm{~s}$ since the administration of MdC. MSCT: multislice computed thomography; CEUS: contrast enhanced ultrasound
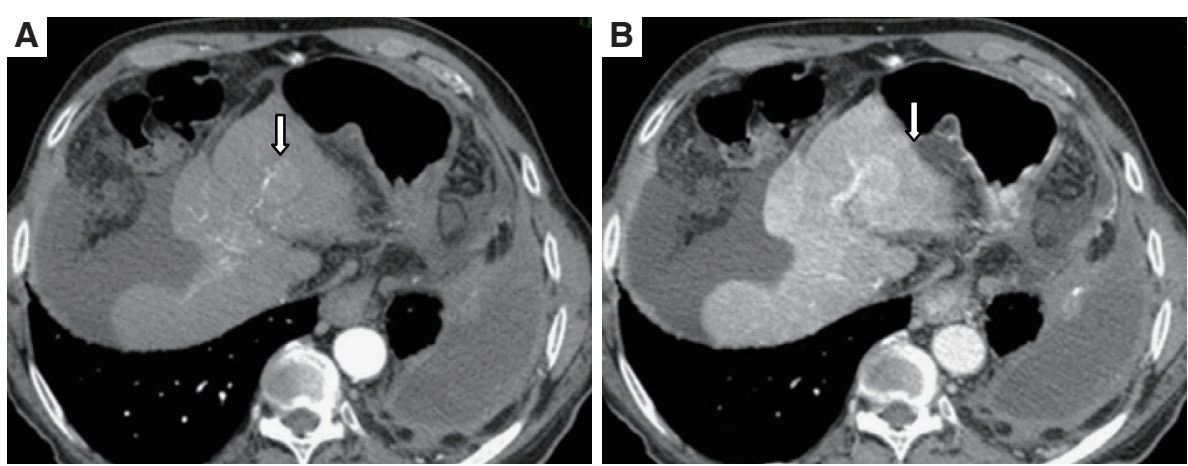

Figure 2: Arterial phase $(A, C)$ and portal phase $(B, D)$ seen in MSCT and CEUS in the same patient with hepatocarcinoma. MSCT (A, B) allows a good documentation of the nodular lesion (arrows) slightly hypervascular in the arterial phase with wash-out in the portal phase. In CEUS the lesion, not detected in B mode, is isovascular in the arterial phase $(C)$; in the porta phase (D) is slightly hypovascular (black arrow head). MSCT: multislice computed thomography; CEUS: contrast enhanced ultrasound
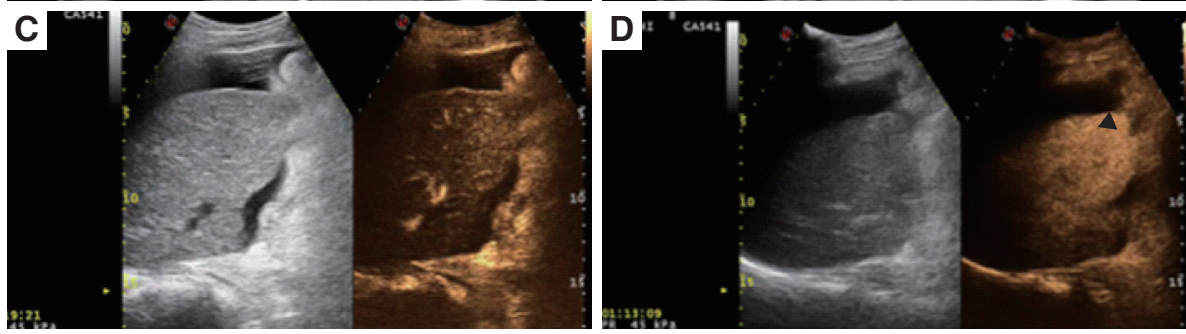

Table 2: Determination of vascularization using CEUS, related to the depth of nodules

\begin{tabular}{lccccc}
\hline \multirow{2}{*}{$\begin{array}{l}\text { Arterial phase } \\
\text { in MSCT }\end{array}$} & $\begin{array}{c}\text { Depth } \\
\text { (cm) }\end{array}$ & \multicolumn{4}{c}{ Early vascular phase seen in CEUS } \\
\hline \multirow{2}{*}{ Hypervascular } & $\leq 9$ & $61(95 \%)$ & $3(5 \%)$ & 64 & 0.0007 \\
& $>9$ & $7(58 \%)$ & $5(42 \%)$ & 12 & \\
Isovascular & $\leq 9$ & $3(43 \%)$ & $4(57 \%)$ & 7 & NS \\
Hypovascular & $>9$ & $1(25 \%)$ & $2(75 \%)$ & 3 & \\
& $>9$ & $2(40 \%)$ & $3(60 \%)$ & 5 & NS \\
& $>9$ & 0 & $1(100 \%)$ & 1 & \\
\hline
\end{tabular}

MSCT: multislice computed thomography; CEUS: contrast enhanced ultrasound; NS: not significant

12 of 76 nodules localized at a depth greater than 9 $\mathrm{cm}$ that appeared hypervascular using MSCT, 7 (58\%) appeared hypervascular using CEUS $(P=0.0007)$.
These results showed that there was a significant difference in the percentage of comparison of the vascularization between the nodules situated at a depth not greater than $9 \mathrm{~cm}$, compared to those studied at a greater depth.

In reference to the size of the lesion, the percentage of vascularization using CEUS in the arterial phase comparing with MSCT was $84 \%$ in lesions with dimensions equal or less than $1 \mathrm{~cm}, 91 \%$ in lesions with dimensions between 1 and $2 \mathrm{~cm}$ and $96 \%$ in lesions greater than $2 \mathrm{~cm}$ [Table 3].

Therefore there was no significant difference in comparing the dimensions of lesions and their vascularization in the arterial phase. 

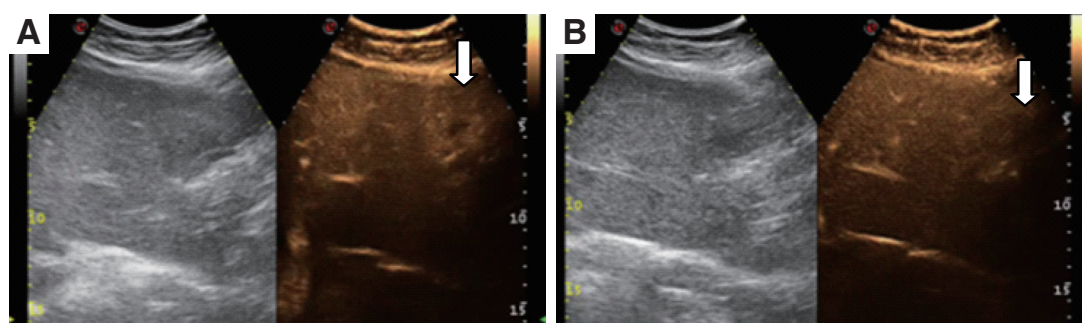

Figure 3: Arterial phase $(A, C)$ and portal phase $(B, D)$ seen in MSCT and CEUS in the same patient with hepatocarcinoma. In CEUS the nodule (arrows) is slightly hypervascular in the arterial phase $(A)$ with wash out in the portal phase (B). The enhancement is disomogeneous becaus of the presence of an avascular area in the cranial portion of the lesion. In MSCT the lesion is isovascluar in the arterial phase (C). In the portal phase (D) it is hypovascular
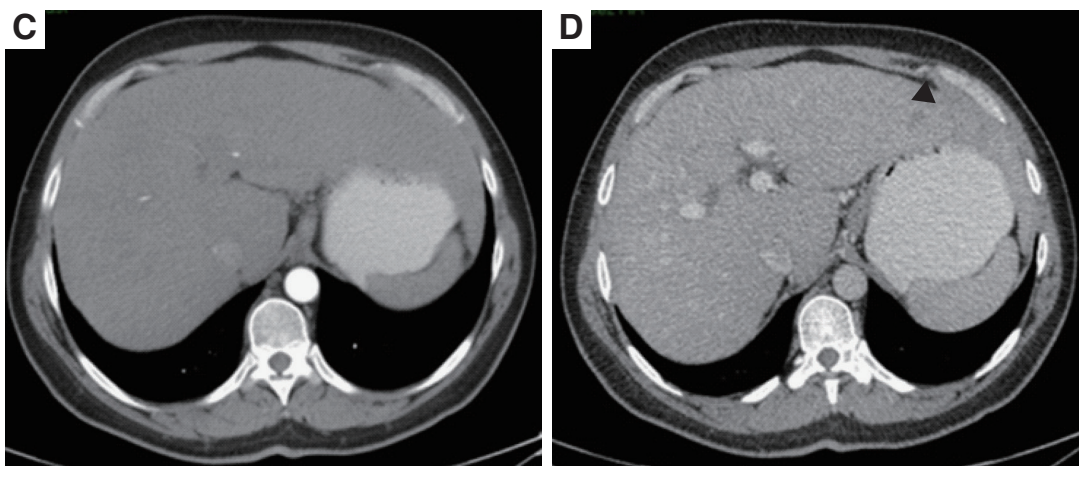
(black arrow head). MSCT: multislice computed thomography; CEUS: contrast enhanced ultrasound

Table 3: Determination of vascularization seen in CEUS related to sizes of lesions

\begin{tabular}{lccccc}
\hline \multirow{2}{*}{$\begin{array}{l}\text { Arterial phase } \\
\text { in MSCT }\end{array}$} & $\begin{array}{c}\text { Sizes } \\
\text { (cm) }\end{array}$ & \multicolumn{4}{c}{ Early vascular phase seen in CEUS } \\
\hline \multirow{4}{*}{ Hypervascular } & $\leq 1$ & $10(84 \%)$ & Iso & Total & $P$-value \\
\hline \multirow{4}{*}{ Isovascular } & $1-2$ & $34(91 \%)$ & $3(9 \%)$ & 37 & NS \\
& $>2$ & $24(96 \%)$ & $1(4 \%)$ & 25 & \\
& $\leq 1$ & $3(60 \%)$ & $2(40 \%)$ & 5 & \\
Hypovascular & $1-2$ & 0 & $6(100 \%)$ & 6 & \\
& $>2$ & 0 & 0 & 0 & \\
& $1-2$ & $1(100 \%)$ & 0 & 1 & \\
& $>2$ & $1(33 \%)$ & $2(67 \%)$ & 3 & \\
\hline
\end{tabular}

MSCT: multislice computed thomography; CEUS: contrast enhanced ultrasound; NS: not significant

Similar results were shown for the portal and sinuisoidal phase [Table 4]: 85 of 92 nodules appeared hypodense using MSCT; using CEUS, 70 nodules $(82 \%)$ were hypovascular, the other $15(18 \%)$ being isovascular. Seven of 92 nodules appeared isodense using MSCT; using CEUS, 6 (86\%) were hypovascular, $1(14 \%)$ was isovascular.

The basic pathology, a chronic liver disease displayed by almost all the examined patients, did not limit significantly the study of intralesional vascularization using CEUS as compared with using CT, particularly in the arterial phase. In the portal phase, the parenchymal enhancement found using CEUS was less intense and more delayed when compared to the enhancement found using CT.

\section{DISCUSSION}

The advent of second-generation echographic contrast
Table 4: Lesions detected in portal phase using CEUS, related to MSCT

\begin{tabular}{lccc}
\hline \multirow{2}{*}{ Portal phase MSCT } & \multicolumn{3}{c}{ Portal phase seen in CEUS } \\
\cline { 2 - 4 } & Iso & Hypo & Total \\
\hline Isodense & $1(14 \%)$ & $6(86 \%)$ & 7 \\
Hypodense & $15(18 \%)$ & $70(82 \%)$ & 85 \\
Total & $16(17 \%)$ & $76(83 \%)$ & 92 \\
\hline
\end{tabular}

MSCT: multislice computed thomography; CEUS: contrast enhanced ultrasound

media and the generation and development of dedicated software allow clinicians, working at a low mechanical index, to study perfusion in real time and also makes easier the study of small-dimension lesions by considerably increasing the diagnostic capabilities of ultrasound. ${ }^{[16-20]}$

In the guidelines for management of HCC provided in 2005 by the American Association for the Study of Liver Disease (AASLD), CEUS has been considered among the non-invasive methods able to detect the typical enhancement of HCC. This condition is characterized by hypervascularization in the arterial phase with progressive wash-out of the MdC in the portal and late phases. ${ }^{[21,22]}$ Such contrastographic characteristics demonstrated high diagnostic validity, in various case studies being characterized by a $92-94 \%$ sensitiveness and by specificity of $87-96 \%$.

The feedback from typical enhancement with the use of CT or magnetic resonance (MR), methods can be considered conclusive for correct diagnosis. Also, $M R$, if performed with hepatospecific MdC (BOPTA and/or EOB), can also allow in late sequences (colongiographic) the demonstration of hepatocyte alteration characterized by hypointensity of signal 
within the lesion, giving further diagnostic evidence of malignancy.

In a multicentric study (DEGUM) with 1,349 patients with focal hepatic lesions identified with basal ultrasound, CEUS was compared with biopsy in $75 \%$ of the cases and in the remaining $25 \%$ with spiral CT or MR. The diagnostic accuracy of CEUS was $90.3 \% .^{[23,24]}$

Two other, more recent prospective studies (DEGUM) have evaluated the potential of CEUS in the characterization of focal hepatic lesions by comparing CEUS with CT and with MR; in both studies it was concluded that there are not statistically significant differences. ${ }^{[25,26]}$

In the first study the authors concluded that CEUS must be used first, before using CT; they have also documented that CEUS utilization can considerably reduce the number of diagnostic biopsies. ${ }^{[25]}$

The second study demonstrated a substantial overlap between the vascularization documented using CEUS when compared with that documented using MR. ${ }^{[26]}$

Gaiani et al. ${ }^{[16]}$ have found that $91 \%$ of hypervascular hepatocarcinoma using MSCT presented hypervascularization in arterial phase with CEUS as well, and that $75 \%$ of hypervascular hepatocarcinoma showed hypovascularization in portal or late phase.

$\mathrm{Xu}$ et al. ${ }^{[19]}$ reported in their series that $87 \%$ of hepatocarcinoma, all with dimensions equal to or less than $2 \mathrm{~cm}$, appeared hypovascular in the portal phase, while $46 \%$ were isovascular in the portal phase.

In this study, a high comparability was demonstrated between CEUS and MSCT, with $88 \%$ of nodules appearing hypervascular in the arterial phase using both methods, independently of lesion dimensions.

Two studies, however, have demonstrated that the sensitivity of CEUS diagnosing $\mathrm{HCC}$ is in direct proportion to lesion dimensions. For the nodules with dimensions equal to or less than $2 \mathrm{~cm}$, Gaiani et al. ${ }^{[16]}$ and Giorgio et al. ${ }^{[20]}$ reported a $83.3 \%$ and $56.3 \%$ sensitivity for CEUS, respectively. Conversely, in nodules with dimensions $>2 \mathrm{~cm}$, sensitivity was significantly increased by $94 \%$ and $91 \%$, for CEUS, in the respective studies.

In this study, there were no statistically significant differences in individualization of vascularization in lesions, in relation to dimensions in the arterial phase. Conversely using CEUS, the evaluation of vascularization in relation to the legion depth was statistically significant. In particular, only $58 \%$ of the lesions situated at a depth greater than $9 \mathrm{~cm}$ from the abdominal wall presented in arterial phase CEUS, the same vascularization as with the corresponding phase in MSCT; this contrasts with $95 \%$ of the lesions situated more superficially.

In this study, the homogeneity of the enhancement was not evaluated because this element can be extremely variable due to a number of factors. Particularly in the arterial phase, inhomogeneity of enhancement is frequently present due to the presence of adipose degeneration or intratumoral necrosis. In the portal phase, a "mosaic" aspect is often noticed, particularly in the larger lesions. ${ }^{[27]}$

The use of CEUS also allows clinicians to differentiate $\mathrm{HCC}$ from other benign or malignant focal hepatic lesions. $^{[28-32]}$

The intrinsic limitations of CEUS vary in relation to various patient characteristics (cooperation,obesity), various characteristics of lesions (site-dimensionsdepth), and the CEUS operator. ${ }^{[33]}$

Another important CEUS limitation is that the technique focuses study on a single lesion, mainly in the arterial phase, because it can often be particularly challenging to evaluate the enhancement of the entire hepatic parenchyma in a short period of time. By contrast, the panoramic views of CT and MR allow scans to evaluate the entire hepatic parenchyma.

In the 2010 AASLD guidelines for the management of HCC, CEUS was removed from the protocol because it can give false positives in patients with intrahepatic cholangiocarcinoma. ${ }^{[34]}$ However, CEUS is the only method that allows the study of the vascularization of a single lesion "in real time". Such a possibility provides the advantage of accurately documenting the neoangiogenesis typical of hepatocarcinoma, characterized by the formation of neoartorioles at the periphery and the inside of the lesion that can be enhanced at a very early stage. Furthermore, in some cases (mainly in small-dimension lesions) such precocity can be transitory and thus assessable only in a continuous view.

Some studies have demonstrated that a certain number of lesions, varying between $5 \%$ and $25 \%$, remain undetermined after a CEUS study, because they do not present a characteristic enhancement. ${ }^{[28-30]}$ This number can be reduced, even if not in a significant manner, if a second method of CT or MR is added to 
CEUS for the study of vascularization.

In our study the sensitiveness, specificity and accuracy of CEUS in the diagnosis of HCC were not evaluated. The intralesional vascularization documented with CEUS compared with that documented with MSCT was respectively compared in a series of hepatic carcinoma. The MR was not documented for comparison because it is not utilized in all cases.

In the event, when using various imaging techniques, when it is not possible to obtain a differential diagnosis between a benign or malignant lesion, it is essential to perform biopsy or monitoring of the patients, depending on the dimensions of the lesion.

In conclusion, this study demonstrates that CEUS is a reliable and accurate method for documenting the intralesional vascularization of hepatic carcinoma, in particular when combined with MSCT. However, CEUS presents some limitations, mainly in relation to the site of lesions.

\section{Financial support and sponsorship None.}

\section{Conflicts of interest}

There are no conflicts of interest.

\section{Patient consent}

Each patient was informed of the study and gave their consent.

\section{Ethics approval}

This study was approved by the Corporate Ethics Committee.

\section{REFERENCES}

1. Tanaka S, Kitamura T, Fujita M, Nakanishi K, Okuda S. Color Doppler flow imaging of liver tumors. AJR Am J Roentgenol 1990;154:509-14.

2. Lencioni R, Pinto F, Armillotta N, Bartolozzi C. Assessment of tumor vascularity in hepatocellular carcinoma: comparison of power Doppler US and color Doppler US. Radiology 1996;201:353-8.

3. Winter TC 3rd, Takayasu K, Muramatsu Y, Furukawa H, Wakao F, Koga H, Sakamoto M, Hirohashi S, Freeny PC. Early advanced hepatocellular carcinoma: evaluation of CT and MR appearance with pathologic correlation. Radiology 1994;192:379-87.

4. Takayasu K, Muramatsu Y, Furukawa H, Wakao F, Moriyama N, Takayama T, Yamasaki S, Sakamoto M, Hirohashi S. Early hepatocellular carcinoma appearance at CT during arterial portography and CT arteriography with pathologic correlation. Radiology 1995;194:101-5.

5. Hayashi M, Matsui O, Ueda K, Kawamori Y, Kadoya M, Yoshikawa J, Gabata T, Takashima T, Nonomura A, Nakanuma Y. Correlation between the blood supply and grade of malignancy of hepatocellular nodules associated with liver cirrosi: evaluation by CT during intrarterial injection of contrast medium. AJR Am J Roentgenol 1999;172:969-76.

6. Stark DD, Weissleder R, Elizondo G, Hahn PF, Saini S, Todd LE, Wittenberg J, Ferrucci JT. Superparamagnetic iron oxide: clinical application as a contrast agent for MR imaging of the liver. Radiology 1998;168:297-301.

7. Imai $\mathrm{Y}$, Murakami T, Yoshida S, Nishikawa M, Ohsawa M, Tokunaga K, Murata M, Shibata K, Zushi S, Kurokawa M, Yonezawa T, Kawata S, Takamura M, Nagano H, Sakon M, Monden M, Wakasa K, Nakamura H. Superparamagnetic iron oxide-enhanced magnetic resonance imaging oh hepatocellular carcinoma: correlation with histological grading. Hepatology 2000;32:205-12.

8. Fujimoto M, Moriyasu F, Nishikawa K, Nada T, Okuma M. Color Doppler sonography of hepatic tumors with a galactose-based contrast agent: correlation with angiographic findings. AJR Am J Roentgenol 1994;163:1099-104.

9. Choi BI, Kim TK, Han JK, Kim AY, Seong CK, Park SJ. Vascularity of hepatocellular carcinoma: assessment with contrast-enhanced second-harmonic versus conventional power Doppler US. Radiology 2000;214:381-6.

10. Lencioni R, Cioni D, Bartolozzi C. Tissue harmonic and contrastspecific imaging: back to gray scale in ultrasound. Eur Radiol 2002;12:151-65.

11. Hirai T, Ohishi H, Tokuno E, Takahashi M, Sakaguchi H, Anai H, Nishimoto Y, Hirohashi S, Kichikawa K. Qualitative diagnosis of hepatocellular carcinoma by contrast-enhanced ultrasonography using coded harmonic angio with Levovist. J Med Ultrason 2002;29:3-9.

12. Koda M, Matsunaga Y, Ueki M. Qualitative assessment of tumor vascularity in hepatocellular carcinoma by contrast-enhanced coded ultrasound: comparison with arterial phase of dynamic CT and conventional color/power Doppler ultrasound. Eur Radiol 2004; $14: 1100-8$

13. Dill-Macky MJ, Burns PN, Khalili K, Wilson SR. Focal hepatic masses: enhancement patterns with SH U 508A and pulse-inversion US. Radiology 2002;222:95-102.

14. Nicolau C, Brú C. Focal liver lesions: evaluation with contrast enhanced ultrasonography. Abdom Imaging 2004;29:348-59.

15. Quaia E, Calliada F, Bertolotto M, Rossi S, Garioni L, Rosa L, Pozzi-Mucelli R. Characterization of focal liver lesions with contrastspecific US modes and a sulfur hexafluoride-filled microbubble contrast agent: diagnostic performance and confidence. Radiology 2004;232:420-30.

16. Gaiani S, Celli N, Piscaglia F, Cecilioni L, Losinno F, Giangregorio F, Mancini M, Pini P, Fornari F, Bolondi L. Usefulness of contrastenhanced perfusionale sonography in the assessment of hepatocellular carcinoma hypervascular at spiral computed tomography. J Hepatol 2004;41:421-6.

17. Xu HX, Liu GJ, Lu MD, Xie XY, Xu ZF, Zheng YL, Liang JY. Characterization of focal liver lesions using contrast-enhanced sonography with a low mechanical index mode and a sulfur hexafluoride-filled microbubble contrast agent. J Clin Ultrasound 2006;34:261-72.

18. Liu GJ, Xu HX, Lu MD, Xie XY, Xu ZF, Zheng YL, Liang JY Enhancement pattern of hepatocellular carcinoma: comparison of real-time contrast-enhanced ultrasound and contrast-enhanced computed tomography. Clin Imaging 2006;30:315-21.

19. Xu HX, Xie XY, Lu MD, Liu GJ, Xu ZF, Zheng YL, Liang JY, Chen LD. Contrast-enhanced sonography in the diagnosis of small hepatocellular carcinoma. J Clin Ultrasound 2006;36:257-66.

20. Giorgio A, Ferraioli G, Tarantino L, de Stefano G, Scala V, Scarano F, Coppola C, Del Viscovo L. Contrast-enhanced sonographic appearance of hepatocellular carcinoma in patients with cirrhosis: 
comparison with contrast-enhanced helical CT appearance. AJR Am J Roentgenol 2004;183:1319-26.

21. Quaia E, Bertolotto M, Dalla Palma L. Characterization of liver hemangiomas with pulse-inversion harmonic imaging. Eur Radiol 2002;12:537-44.

22. Bruix J, Sherman M; Practice Guidelines Committee, American Association for the Study of Liver Diseases. Mangement of heptocellular carcinoma. Hepatology 2005;42:1208-36.

23. Strobel D, Seitz K, Blank W, Schuler A, Dietrich C, von Herbay A, Friedrich-Rust M, Kunze G, Becker D, Will U, Kratzer W, Albert FW, Pachmann C, Dirks K, Strunk H, Greis C, Bernatik T. Contrastenhanced ultrasound for the characterization of focal liver lesions-diagnostic accuracy in clinical practice (DEGUM multicenter trial). Ultraschall Med 2008;29:499-505.

24. Strobel D, Seitz K, Blank W, Schuler A, Dietrich CF, von Herbay A, Friedrich-Rust M, Bernatik T. Tumor-specific vascularization pattern of liver metastasis, hepatocellular carcinoma, hemangioma and focal nodular hyperplasia in the differential diagnosis of 1,349 liver lesions in contrast-enhanced ultrasound (CEUS). Ultraschall Med 2009;30:376-82

25. Seitz K, Strobel D, Bernatik T, Blank W, Friedrich-Rust M, Herbay Av, Dietrich CF, Strunk H, Kratzer W, Schuler A. ContrastEnhanced Ultrasound (CEUS) for the characterization of focal liver lesions - prospective comparison in clinical practice: CEUS vs. CT (DEGUM multicenter trial). Parts of this manuscript were presented at the Ultrasound Dreiländertreffen 2008, Davvos. Ultraschall Med 2009;30:383-9.

26. Seitz K, Bernatik T, Strobel D, Blank W, Friedrich-Rust M, Strunk H, Greis C, Kratzer W, Schuler A. Contrast-enhanced ultrasound (CEUS) for the characterization of focal liver lesions in clinical practice (DEGUM Multicenter Trial): CEUS vs. MRI--a prospective comparison in 269 patients. Ultraschall Med 2010;31:492-9.
27. Catalano O, Nunziata A, Lobianco R, Siani A. Real-time harmonic contrast material-specific US of focal liver lesions. Radiographics 2005;25:333-49.

28. Loria F, Loria G, Basile S, Crea G, Frosina L, Di Carlo I. Contrastenhanced ultrasound appearance of enhancement patterns of intrahepatic cholangiocarcinoma: correlation with pathological findings. Updates Surg 2014;66:135-43.

29. Toro A, Mahfouz AE, Ardiri A, Malaguarnera M, Malaguarnera G, Loria F, Bertino G, Di Carlo I. What is changing in indications and treatment of hepatic hemangiomas. A review. Ann Hepatol 2014;13:327-39.

30. Von herbay A, Westendorff J, Gregor M. Contrast-enhanced ultrasound with sonovue: differentiation between benign and malignant focal liver lesions in 317 patients. J Clin Ultrasound 2010;38:1-9.

31. Sirli R, Sporea I, Martie A, Popescu A, Dănilă M. Contrast-enhanced ultrasound in focal liver lesions- a cost efficiency study. Med Ultrason 2010;12:280-5 .

32. Bryant TH, Blomley MJ, Albrecht T, Sidhu PS, Leen EL, Basilico R, Pilcher JM, Bushby LH, Hoffmann CW, Harvey CJ, Lynch M, MacQuarrie J, Paul D, Cosgrove DO. Improved characterization of liver lesions with liver-phase uptake of liver specific microbubbles: prospective multi center trial. Radiology 2004;232:799-809.

33. Loria F, Loria G, Basile S, Crea G, Randazzo D, Frosina L. Contrastenhanced ultrasound of hepatocellular carcinoma: correlation between enhancement pattern and cellular differentiation on histopatology. Updates Surg 2012;64:247-55.

34. Galassi M, Iavarone M, Rossi S, Bota S, Vavassori S, Rosa L, Leoni S, Venerandi L, Marinelli S, Sangiovanni A, Veronese L, Fraquelli M, Granito A, Golfieri R, Colombo M, Bolondi L, Piscaglia F. Patterns of appearance and risk of misdiagnosis of intrahepatic cholangiocarcinoma in cirrosi at contrast enhanced ultrasound. Liver Int 2013;33:771-9. 